\title{
The $\alpha$-shape Based Natural Element Method in Solid and Fluid Mechanics
}

\author{
D. González, I. Alfaro, E. Cueto, M. Doblaré, and F. Chinesta
}

1 Group of Structural Mechanics and Material Modelling. Aragón Institute of Engineering Research (I3A). University of Zaragoza. María de Luna, 3. E-50018 Zaragoza, Spain.

2 Laboratoire de mécanique des systèmes et des procédés. LMSP UMR 8106 CNRS-ENSAM-ESEM. 151 Bd. de l'Hôpital, 75013 Paris, France.

\begin{abstract}
The Natural Element method (also known as Natural Neighbour Galerkin method) is a Galerkin method based on the use of Natural Neighbour interpolation to construct the trial and test functions. Unlike many other meshless methods, it has some important characteristics, such as interpolant shape functions, easy imposition of essential boundary conditions and linear precision along convex boundaries.

The natural neighbour interpolation scheme is based on the construction of a Delaunay triangulation of the given set of points. This geometrical link provides the NEM some other interesting properties. One of them is the ability of constructing models without any explicit (CAD) boundary description. Instead, by invoking the concept of $\alpha$-shape of the cloud of points, the method is able to accurately extract the geometry described by the nodes as it evolves, thus avoiding complex geometrical checks in the formation of holes or waves in the domain, without any loss in mass conservation requirements. It has been also proved how the use of $\alpha$-shapes ensures the strictly interpolant character of the shape functions along any type of boundary. In this work we review the main characteristics of the method in its application to Solid and Fluid Mechanics, including the study of mixed natural neighbour approximation, simulation of nearly incompressible media and some industrial applications.
\end{abstract}

\section{Introduction}

In this work we analyse the main features of one member of the wide family of meshless methods. Natural Neighbour Galerkin methods (also known as Natural Element methods, NEM) are based on the use of any natural neighbour-based interpolation scheme. The most extended of these schemes is often referred to as Sibson interpolation [18]. Among the most remarkable properties of the resulting methods is the strictly interpolant character of the resulting approximation, leading to an easy imposition of essential (Dirichlet) boundary conditions. Also, the fact that the computation of the shape functions is made - alt least, formally - upon the Delaunay triangulation of 
the cloud of points, confers the method with a sound geometrical basis. This allows, form instance, to couple the method with some geometrical methods, such as $\alpha$-shapes [12]. This will allow to build a method in which the geometry of the domain is extracted as it evolves, without any geometrical description of the boundary. Thus, boundary evolution, with holes or waves formation, is naturally handled by the method in a very efficient way.

The resulting method appears to be specially well suited to be used in an updated Lagrangian framework, since it has been proved that the shape of Delaunay triangles does not affect the quality of the results [19]. In this work, we review the main characteristics of the method when applied to Fluid and Solid Mechanics with large strains. In section 2 we revisit the basics of natural neighbour interpolation. In section 3 we review the governing equations for incompressible media, together with the conditions that ensure stable approximations (LBB condition) and suitable enriched NE approximations. In section 4 we review the $\alpha$-shape based Natural Element Method, firstly proposed in [9] and its potential applications in free surface flows. Finally, in section 5 two examples corresponding to Solid and Fluid Mechanics are presented.

\section{Natural Neighbour Galerkin Methods}

\subsection{Natural Neighbour Interpolation}

Natural neighbour interpolation is a method to interpolate multivariate data first established by Sibson [18]. Recently, it has been generalised in [5] and [15]. It relies in the concepts of Voronoi diagram [22] of the given cloud of points and its dual structure, the Delaunay triangulation [11]. The Delaunay triangulation (tetrahedrisation) of a cloud of points $N=\left\{n_{1}, \ldots, n_{N}\right\} \in \mathbb{R}^{2}$ $\left(\mathbb{R}^{3}\right)$ is the decomposition of the convex hull of the points into $k$-simplexes (where $k$ represents the dimension of the simplex, that is, $k=2$ for a triangle and $k=3$ for a tetrahedron) such that the empty circumcircle criterion holds. That is, the circumcircle (circumsphere) of each simplex contains no other point of the cloud $N$. Fig. 2.1 represents the Delaunay triangulation of a cloud of points.

For a given node $n_{I}$, the associated Voronoi cell is composed by all of the points which are closer to the node $n_{I}$ than to any other node. Formally,

$$
T_{I}=\left\{\boldsymbol{x} \in \mathbb{R}^{n}: d\left(\boldsymbol{x}, \boldsymbol{x}_{I}\right)<d\left(\boldsymbol{x}, \boldsymbol{x}_{J}\right) \forall J \neq I\right\}
$$

where $T_{I}$ is the Voronoi cell and $d(\cdot, \cdot)$ represents the Euclidean distance. In the problems considered in this paper, $n=2,3$.

For the definition of Sibson interpolation it is necessary to previously introduce the concept of second order Voronoi cell. It is defined as the locus of the points that have the node $n_{I}$ as the closest node and the node $n_{J}$ as the second closest node: 

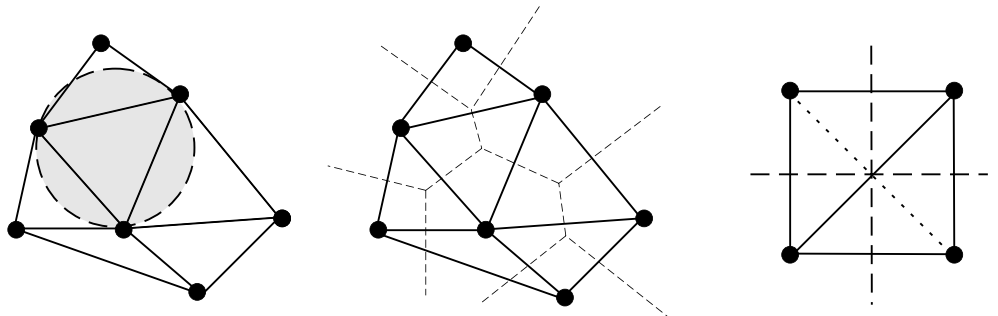

Figure 2.1. Delaunay triangulation and Voronoi diagram of a cloud of points. On the right, an example of a degenerate distribution of nodes, with the two possible triangulations depicted. In this last case, four points lie in the same circumcircle and thus no single triangulation exists.

$$
T_{I J}=\left\{\boldsymbol{x} \in \mathbb{R}^{n}: d\left(\boldsymbol{x}, \boldsymbol{x}_{I}\right)<d\left(\boldsymbol{x}, \boldsymbol{x}_{J}\right)<d\left(\boldsymbol{x}, \boldsymbol{x}_{K}\right) \forall J \neq I \neq K\right\}
$$

If a new node is added to a given cloud of points, the Voronoi cells will be modified by the presence of the new point. Sibson [18] defined the natural neighbour coordinates of a point $\boldsymbol{x}$ with respect to one of its neighbours $I$ as the ratio of the cell $T_{I}$ that is transferred to $T_{x}$, when adding $\boldsymbol{x}$ to the initial cloud of points, to the total area of $T_{x}$. In other words, being $\kappa(\boldsymbol{x})$ and $\kappa_{I}(\boldsymbol{x})$ the Lebesgue measures of $T_{x}$ and $T_{x I}$ respectively, the natural neighbour coordinates of $\boldsymbol{x}$ with respect to the node $I$ is defined as

$$
\phi_{I}^{s i b}(\boldsymbol{x})=\frac{\kappa_{I}(\boldsymbol{x})}{\kappa(\boldsymbol{x})} .
$$

The resultant shape function depends obviously of the relative position of the nodes. An example for a node surrounded by other eight on a regular lattice is depicted in Fig. 2.3.

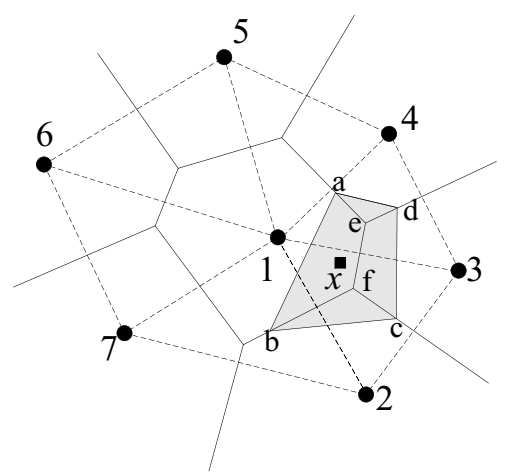

Figure 2.2. Definition of the Natural Neighbour coordinates of a point $\boldsymbol{x}$. 


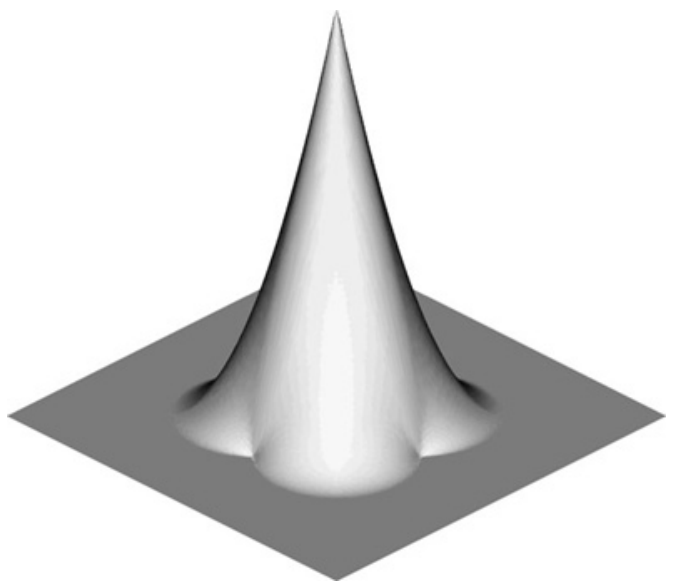

Figure 2.3. Natural Element shape function (courtesy N. Sukumar).

The resultant shape function has some remarkable properties (see [19] or [10] for more in-deep explanations and rigorous proofs of this behaviour). Firstly, it is smooth ( $C^{1}$ at least) everywhere except at the nodes, as can be seen in Fig. 2.3. Natural neighbour (Sibson) shape functions posses linear completeness [19] and form a partition of unity. Therefore, it is possible to enrich natural neighbour interpolants in order to increase the order of the polinomial of the interpolation, as proposed in [3].

\section{Natural Element Methods for Incompressible Media}

The governing equations for incompressible Solid or Fluid Mechanics are:

1. Equilibrium equations (balance of linear momentum in the absence of inertial and body forces):

$$
\boldsymbol{\nabla} \cdot \boldsymbol{\sigma}=\mathbf{0} \text { in } \Omega \text {. }
$$

2. Incompressibility of the medium:

$$
\boldsymbol{\nabla} \cdot \boldsymbol{u}=0 \text { in } \Omega
$$

where $\boldsymbol{\sigma}$ represents the Cauchy stress tensor and $\boldsymbol{u}$ the displacement vector if we deal with Solid Mechanics and usually the velocity vector if it is the case with Fluid Mechanics. Boundary conditions are of the type

$$
\begin{gathered}
\boldsymbol{\sigma} \cdot \boldsymbol{n}=\overline{\boldsymbol{t}} \text { on } \Gamma_{t} \\
\boldsymbol{u}=\overline{\boldsymbol{u}} \text { on } \Gamma_{u} .
\end{gathered}
$$


in which $\boldsymbol{n}$ is the outward normal on the boundary $\Gamma=\Gamma_{u} \cup \Gamma_{t}$, with $\Gamma_{u} \cap \Gamma_{t}=\emptyset$.

If we assume small displacements and strains, the constitutive equations can be expressed, both for an isotropic elastic solid and a newtonian fluid, as

$$
\begin{array}{r}
\boldsymbol{\sigma}=-p \boldsymbol{I}+2 \mu \nabla_{s} \boldsymbol{u} \\
0=\nabla \cdot \boldsymbol{u}-\frac{p}{\lambda}
\end{array}
$$

where $p$ represents the hydrostatic pressure and $\nabla_{s}$ the symmetric part of the gradient operator, thus leading to the Cauchy small strains tensor or to the strain rate tensor in Solid or Fluid Mechanics, respectively. The Lamé parameters $\lambda$ and $\mu$ are expressed in terms of the Young's modulus $E$ and the Poisson's ratio $\nu$ as

$$
\mu=\frac{E}{2(1+\nu)}, \quad \lambda=\frac{2 \mu \nu}{1-2 \nu}
$$

As $\nu$ approaches 0.5 , it is clear that $\lambda$ becomes unbounded, so that the equation (3.6) represents the incompressibility restraint $\nabla \cdot \boldsymbol{u}=0$.

The variational (weak) formulation is usually stated as:

Find $\boldsymbol{u} \in \mathcal{U}$ such that

$$
\begin{gathered}
\int_{\Omega(t)} \boldsymbol{\sigma}(\boldsymbol{u}): \varepsilon^{*} d \Omega=\int_{\Gamma_{t}} \overline{\boldsymbol{t}} \cdot \boldsymbol{u}^{*} d \Gamma \quad \forall \boldsymbol{u}^{*} \in \mathcal{V} \\
\int_{\Omega(t)}\left(-\nabla \boldsymbol{u}+\frac{1}{\lambda} p\right) p^{*} d \Omega=0 \quad \forall p^{*} \in L_{2}(\Omega(t)),
\end{gathered}
$$

where $\mathcal{U}=\left\{\boldsymbol{u}\left|\boldsymbol{u} \in\left(H^{1}(\Omega)\right)^{2}, \boldsymbol{u}\right|_{\Gamma_{u}}=\overline{\boldsymbol{u}}\right\}, \mathcal{V}=\left\{\boldsymbol{u}^{*}\left|\boldsymbol{u}^{*} \in\left(H^{1}(\Omega)\right)^{2}, \boldsymbol{u}\right|_{\Gamma_{u}}=\right.$ $\mathbf{0}\}$, and $\Gamma_{u}$ and $\Gamma_{t}$ are the portions of the boundary of the domain $\Omega$ with prescribed displacements (velocities) and tractions, respectively. $\overline{\boldsymbol{t}}$ and $\overline{\boldsymbol{u}}$ represent such tractions and displacements. As usual, $H^{1}$ and $L_{2}$ are the Sobolev and Lebesgue functional spaces, respectively.

If we approximate the displacement (velocities) and pressures by employing a finite-dimensional set of basis functions, we arrive to a discrete form of the previous equations (Bubnov-Galerkin method)

$$
\begin{aligned}
& \boldsymbol{u}^{h}(\boldsymbol{x})=\sum_{I=1}^{n} \phi_{I}(\boldsymbol{x}) \boldsymbol{u}_{I} \\
& p^{h}(\boldsymbol{x})=\sum_{I=1}^{n} \psi_{I}(\boldsymbol{x}) p_{I} .
\end{aligned}
$$

The functions $\psi_{I}(\boldsymbol{x})$ and $\phi_{I}(\boldsymbol{x})$ in this work represent some form of natural neighbour interpolation, as presented before. This leads to the following system of algebraic equations:

$$
\left(\begin{array}{cc}
\bar{K} & G \\
G^{T} & M
\end{array}\right)\left(\begin{array}{l}
u \\
p
\end{array}\right)=\left(\begin{array}{l}
f \\
0
\end{array}\right)
$$


where

$$
\begin{array}{r}
\overline{\boldsymbol{K}}_{I J}=\int_{\Omega} \boldsymbol{B}_{I}^{T} \overline{\boldsymbol{C}} \boldsymbol{B}_{J} d \Omega \\
\boldsymbol{G}_{I J}=-\int_{\Omega} \tilde{\boldsymbol{B}}_{I}^{T} \psi_{J} d \Omega \\
\boldsymbol{M}_{I J}=-\frac{1}{\lambda} \int_{\Omega} \psi_{I} \psi_{J} d \Omega \\
\boldsymbol{f}_{I}=\int_{\Gamma_{t}} \phi_{I} \overline{\boldsymbol{t}} d \Gamma
\end{array}
$$

and

$$
\begin{array}{r}
\tilde{\boldsymbol{B}}_{I}=\left[\begin{array}{ll}
\phi_{I, 1}(\boldsymbol{x}) & \phi_{I, 2}(\boldsymbol{x})
\end{array}\right] \\
\boldsymbol{B}_{I}=\left(\begin{array}{cc}
\phi_{I, 1}(\boldsymbol{x}) & 0 \\
0 & \phi_{I, 2}(\boldsymbol{x}) \\
\phi_{I, 2}(\boldsymbol{x}) & \phi_{I, 1}(\boldsymbol{x})
\end{array}\right) \\
\bar{C}_{I J K L}=\mu\left(\delta_{I K} \delta_{J L}+\delta_{I L} \delta_{J K}\right)
\end{array}
$$

It must be noted that, if we consider totally incompressible situations, $\boldsymbol{M}=\mathbf{0}$.

As it is well known, not all of the displacement-pressure approximations constructed in this way lead to stable and convergent results [4]. The conditions to be fulfilled by the chosen approximation are determined by the inf-sup or Ladyzhenskaya-Babuška-Brezzi (LBB) condition [2] [7], together with the elipticity condition of the resultant formulation. The LBB condition may be written as:

$$
\inf _{p^{h} \in \mathcal{P}_{h}} \sup _{\boldsymbol{u}^{h} \in \mathcal{U}_{h}} \frac{\int_{\Omega} p^{h} \operatorname{div} \boldsymbol{u}^{h} d \Omega}{\left\|p^{h}\right\|_{0}\left\|\boldsymbol{u}^{h}\right\|_{1}}=\gamma_{h} \geq \gamma>0
$$

where $\gamma$ is a positive constant independent of the mesh size, $h . \mathcal{P}_{h}$ and $\mathcal{U}_{h}$ represent the pressure and displacement approximation spaces.

This condition is rarely proved analytically. Instead, its fulfillment is usually checked numerically. In [14], the authors have tested some mixed formulations arising from the Partition of Unity enrichment [3] of the displacement (or velocity) field. If the resultant approximation is able to reproduce the $x y$ monomial, together with the usual NE linear consistency, it seems to verify the LBB condition. The resultant shape functions are depicted in Fig. 3.4.

In order to verify the fulfilment of the LBB condition, Bathe [4] proposed a numerical test based on the use of a finite, small, set of meshes. To evaluate expression (3.20), an equivalent discrete form is developed:

$$
\inf _{\boldsymbol{W}_{h}} \sup _{\boldsymbol{U}_{h}} \frac{\boldsymbol{W}_{h}^{T} \boldsymbol{G}_{h} \boldsymbol{U}_{h}}{\sqrt{\boldsymbol{W}_{h}^{T} \boldsymbol{G}_{h} \boldsymbol{W}_{h}} \cdot \sqrt{\boldsymbol{U}_{h}^{T} \boldsymbol{S}_{h} \boldsymbol{U}_{h}}}=\gamma_{h} \geq \gamma>0
$$




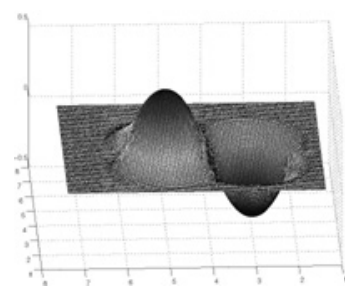

(a)

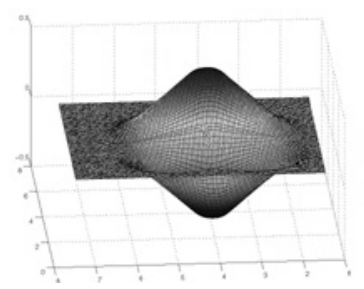

(b)

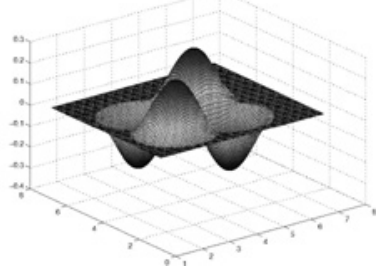

(c)

Figure 3.4. Plot of the shape functions $\phi x$ (a), $\phi y$ (b) and $\phi x y$ (c).

where $\boldsymbol{W}_{h}$ and $\boldsymbol{U}_{h}$ are vectors of nodal values corresponding to pressures and displacements, respectively. $\boldsymbol{G}_{h}$ and $\boldsymbol{S}_{h}$ are the matrices associated with the norms

$$
\left\|p_{h}\right\|_{0}^{2}=\boldsymbol{W}_{h}^{T} \boldsymbol{G}_{h} \boldsymbol{W}_{h} \text { and }\left\|\boldsymbol{u}_{h}\right\|_{1}^{2}=\boldsymbol{U}_{h}^{T} \boldsymbol{S}_{h} \boldsymbol{U}_{h}
$$

$\boldsymbol{G}_{h}$ and $\boldsymbol{S}_{h}$ are positive semidefinite and positive definite [8], respectively. It is then demonstrated that the first non-zero eigenvalue, $\lambda_{k}$, of the problem

$$
\boldsymbol{G}_{h} \boldsymbol{\phi}_{h}=\lambda \boldsymbol{S}_{h} \boldsymbol{\phi}_{h}
$$

is related to the searched value $\gamma_{h}$ through the expression

$$
\gamma_{h}=\sqrt{\lambda_{k}} .
$$

If the constructed approximation for a given problem consists of $n_{p}$ pressure degrees of freedom and $n_{u}$ displacement degrees of freedom, the number of spurious pressure modes is given by

$$
k_{p m}=k-\left(n_{u}-n_{p}-1\right)
$$

Bathe [4] proposed the use of sequences composed of three or more meshes in order to test a given approximation. If the $\gamma_{h}$ value is not bounded away from 0 , one can say that the LBB condition is not satisfied. The test is only valid for the given geometry and problem considered, but it can be assured that if the test is not passed, the approximation will not verify the LBB condition.

In order to test the ability of the proposed formulations to pass the inf-sup condition, we have checked a sequence of three meshes, composed by $3 \times 3$, $4 \times 4$ and $5 \times 5$ nodes, regularly and irregularly distributed over a square of side unity (see Fig. 3.5).

Results for these discretisations are shown in Fig. 3.6. Results for the test applied over the 3/1 triangular Finite Element are also shown. It can be seen how the Sibson approximation, enriched with $\{1, x, y, x y\}$, for the displacements gives good results, both using Thiessen $\left(\mathcal{C}^{-1}\right)$ and Sibson interpolations 


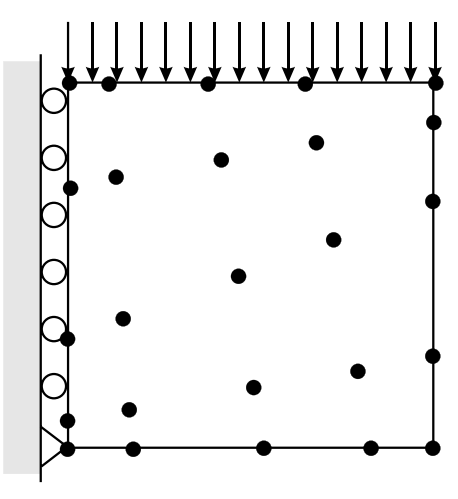

Figure 3.5. Geometry of the problem used to evaluate the numerical inf-sup test.

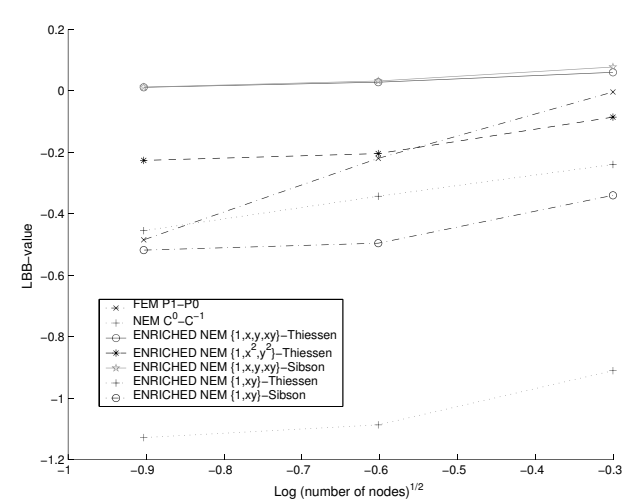

Figure 3.6. Inf-sup test for the proposed approximations.

for the pressure. However, the enrichment with the monomials $\left\{1, x^{2}, y^{2}\right\}$, initially proposed in order to avoid rank deficiency in the resultant stiffness matrix, gives rise to spurious pressure modes. These same spurious modes can be obtained for certain configurations in the Sibson-Thiessen element, in a way similar to the bilinear displacement-constant pressure quadrilateral Finite Element [4].

Although results for a unique problem are not extrapolable, the proposed formulation for the $\{1, x, y, x y\}$ enrichment seems to be stable and adequate for a wide variety of problems. Also, enrichment with the set $\{1, x y\}$ seems to give stable approximations. In this case, the resulting approximation closely resembles the MINI element [1] (linear triangular finite element with bubble and discontinuous approximation for pressure).

The most usual Sibson-Thiessen mixed approximation closely resembles the bilinear displacement-constant pressure finite element, which presents spurious modes under certain boundary conditions. However, for most practical applications, it has shown a good behaviour and has never presented locking. An example is presented in section 5.2 in which this approximation is used.

\section{The $\alpha$-shape based Natural Element Method}

In most Lagrangian Fluid Mechanics simulations the tracking of the free surfaces requires a special treatment, since the possibility of development of holes, waves, etc. exists. Traditionally, this task has been accomplished by discretising the boundary and performing complex geometrical checks. This may include the checking for new free surface boundary segments, if holes are developing, or boundary segments deletion, if the free surface disappears.

In this work we have chosen a different approach, based on the geometrical concept of $\alpha$-shapes [13]. In addition, the authors have shown that the 
construction of natural neighbour-based interpolants over an $\alpha$-shape of the domain leads to linearly interpolant approximations along the whole boundary, thus solving one of the biggest problems of meshless methods. The problem can be formulated briefly as follows: can the cloud of points itself contain enough information about the geometry of the domain over which it is defined? If the density of the cloud is enough, the answer is affirmative.

An $\alpha$-shape is a polytope that is not necessarily convex nor connected, being triangulated by a subset of the Delaunay triangulation of the points. Thus, the empty circumcircle criterion holds. Let $N$ be a finite set of points in $\mathbb{R}^{3}$ and $\alpha$ a real number, with $0 \leq \alpha<\infty$. A $k$-simplex $\sigma_{T}$ with $0 \leq k \leq 3$ is defined as the convex hull of a subset $T \subseteq N$ of size $|T|=k+1$. Let $b$ be an $\alpha$-ball, that is, an open ball of radius $\alpha$. A $k$-simplex $\sigma_{T}$ is said to be $\alpha$-exposed if there exist an empty $\alpha$-ball $b$ with $T=\partial b \bigcap N$ where $\partial$ means the boundary of the ball. In other words, a $k$-simplex is said to be $\alpha$-exposed if an $\alpha$-ball that passes through its defining points contains no other point of the set $N$.

Following this, we can define the family of sets $F_{k, \alpha}$ as the sets of $\alpha$-exposed $k$-simplexes for the given set $N$. This allows us to define an $\alpha$-shape of the set $N$ as the polytope whose boundary consists on the triangles in $F_{2, \alpha}$, the edges in $F_{1, \alpha}$ and the vertices or nodes in $F_{0, \alpha}$. As remarked before, an $\alpha$-shape is a polytope that can be triangulated by a subset of the Delaunay triangulation or tetrahedrization, that is, by an $\alpha$-complex.

In the case of non-uniform nodal distributions, the possibility of using density-scaled $\alpha$-shapes [20] has also been studied in [9]. The resultant geometry of the domain ranges from the cloud of points itself for $\alpha=0$ to the convex hull of the cloud for $\alpha=\infty$. The parameter $\alpha$ can be seen as a measure of the level of detail up to which the domain is represented. If there exists a sufficiently dense nodal sampling it would be easy to find an $\alpha$ value that gives an accurate geometry definition. See $[9,17]$ for a deeper discussion on how the value of $\alpha$ can affect the results.

In this approach, the geometry of the domain is extracted at each time step, with no need of complex geometrical checks. See example 5.2 for an application of the method in an extrusion flow.

\section{Numerical Examples}

\subsection{Cantilever Beam under Bending}

In this section we study the problem of a two-dimensional beam subjected to a parabolically distributed load at its end and fixed at the other side, as shown in Fig. 5.7. We consider a discretisation composed of 85 nodes, as shown in Fig. 5.8.

Material characteristics are: Young's modulus 1.0 and variable Poisson's ratio, ranging from 0.4 to 0.4999999 . To test the performance of the proposed 


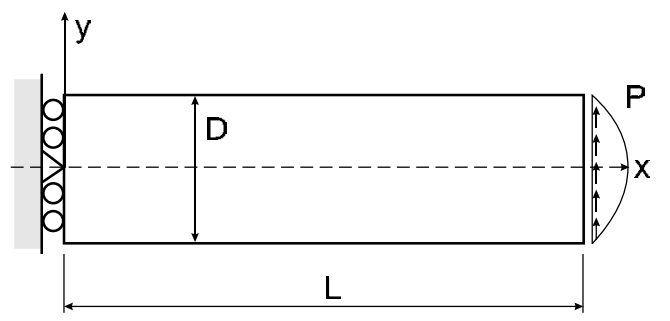

Figure 5.7. Geometry of the cantilever beam under bending.

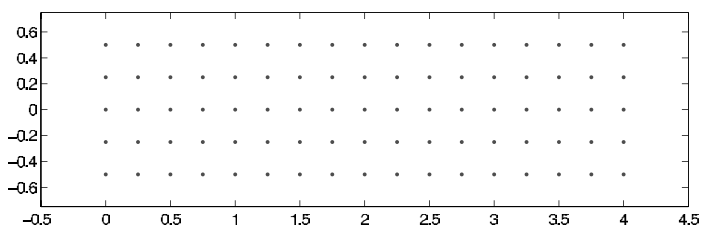

Figure 5.8. Cloud of points for the simulation of a beam under bending.

formulations, we have compared the tip displacement to the theoretical one [21]. In this case, $L=4.0$ and $D=1.0$.

In table 5.1, normalised end displacements $\left(u_{y}(L, 0)\right)$ are presented. These results include those of the FEM, obtained by using the same Delaunay triangles for the construction of the approximation. It can be seen how the displacement-based NEM presents severe locking as the Poisson's ratio increases, as expected. However, all the other constructed approximations present good agreement with the analytical result. In particular, we would like to highlight the good behaviour of the Sibson-Thiessen approximation, previously used by the authors in Fluid Mechanics simulations in [17]. Although it is possible to impose certain boundary conditions so as to generate a spurious pressure mode (in a similar way than to the 4/1 Finite Element [4]), no spurious pressure modes have been observed throughout our computations.

\begin{tabular}{|c|c|c|c|}
\hline Poisson's Ratio & 0.4 & 0.4999 & 0.4999999 \\
\hline \hline FEM-displacement-based & 93.74 & 18.73 & 17.75 \\
\hline FEM-3/3 & 96.38 & 94.45 & 94.45 \\
\hline FEM-3/1 & 100.61 & 101.52 & 101.52 \\
\hline NEM-displacement-based & 94.36 & 19.09 & 19.58 \\
\hline NEM-Sibson-Sibson & 96.78 & 94.93 & 94.94 \\
\hline NEM-Sibson-Thiessen & 99.28 & 99.07 & 99.07 \\
\hline NEM Sibson $\times\left\{1, x^{2}, y^{2}\right\}$-Thiessen & 100.38 & 100.33 & 99.34 \\
\hline NEM Sibson $\times\{1, x, y, x y\}$-Sibson & 99.32 & 100.52 & 100.52 \\
\hline NEM Sibson $\times\{1, x, y, x y\}$-Thiessen & 99.4 & 100.7 & 100.7 \\
\hline
\end{tabular}

Table 5.1. Results for the beam under bending problem, expressed as $\%$ of the theoretical result at the beam end. 
Results are even better for the enriched Sibson approximation. None of the implemented enrichments showed spurious pressure modes for this problem and the obtained accuracy is remarkable. However, the use of enriched approximations is not so intuitive, since the nodal parameters in the discrete system of equations do not represent the nodal displacements.

\subsection{Simulation of Extrusion Processes}

In this section we deal with the simulation of extrusion processes. We consider a benchmark example of an extrusion die whose geometry is shown in Fig. 5.9. For the simulation, only the region of the metal nearest to the die was considered by enforcing appropriate displacements. The cloud of points is composed by 2989 nodes and remained unchanged throughout the simulation. The extruded metal was simulated as viscoplastic, with constitutive equations as follows:

$$
\boldsymbol{\sigma}=-p \boldsymbol{I}+2 \mu(\boldsymbol{D}) \boldsymbol{D}
$$

If we assume a Norton-Hoff plasticity model (viscoplastic flow with null yield stress), viscosity is a function of the second invariant of the strain rate tensor, namely

$$
\mu(\boldsymbol{D})=\mu_{0}(\sqrt{2 \boldsymbol{D}: \boldsymbol{D}})^{n-1}
$$

being $\mu_{0}$ the so-called consistency coefficient and $n$ the pseudo-plasticity coefficient. In this example, the consistency coefficient was $\mu_{0}=1.0 \mathrm{MPa} \cdot \mathrm{s}$ and the pseudo-plasticity $n=0.3$. Note the highly non-linear character of the resultant behaviour. Similar material behaviour was employed in [6] in the simulation of metal forging processes with Corrected Smooth Particle Hydrodynamics methods (CSPH).
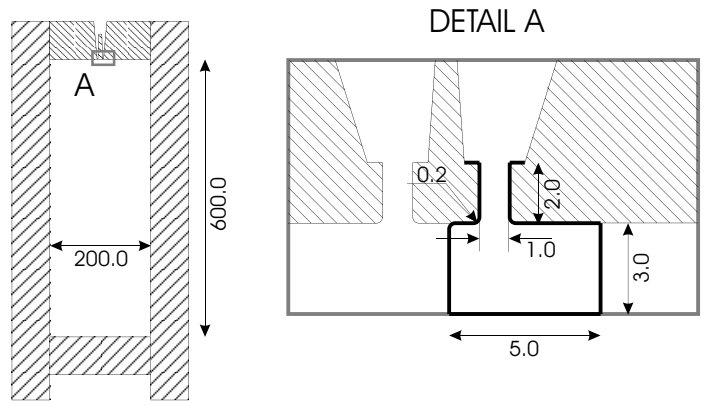

Figure 5.9. Geometry of the extrusion die and detail of the simulated region.

The mould is considered as perfectly rigid and slipping contact between metal and die was assumed. Of course, this model is a first attempt to validate the ability of natural neighbour Galerkin methods to handle such type of 
processes. The springback of the extruded metal can not be predicted, for instance, by assuming a Norton-Hoff plasticity model like the one exposed in Eq. (5.1).

Equivalent plastic strain (or, equivalently, second invariant of the strain rate tensor, $\sqrt{2 \boldsymbol{D}: \boldsymbol{D}}$ ) for time steps 1, 100 and 200 is depicted in Fig. 5.10. It can be noticed the accuracy obtained in the volume prediction (see Fig. 5.11). In this case, total volume error is under $0.25 \%$, much lower than those predicted in references like [16] by using ALE techniques and a similar number of nodes. This is in spite of the high distortion of the Delaunay triangles achieved throughout the simulation (see the $\alpha$-shape of the cloud of points in an intermediate time step in Fig. 5.12).
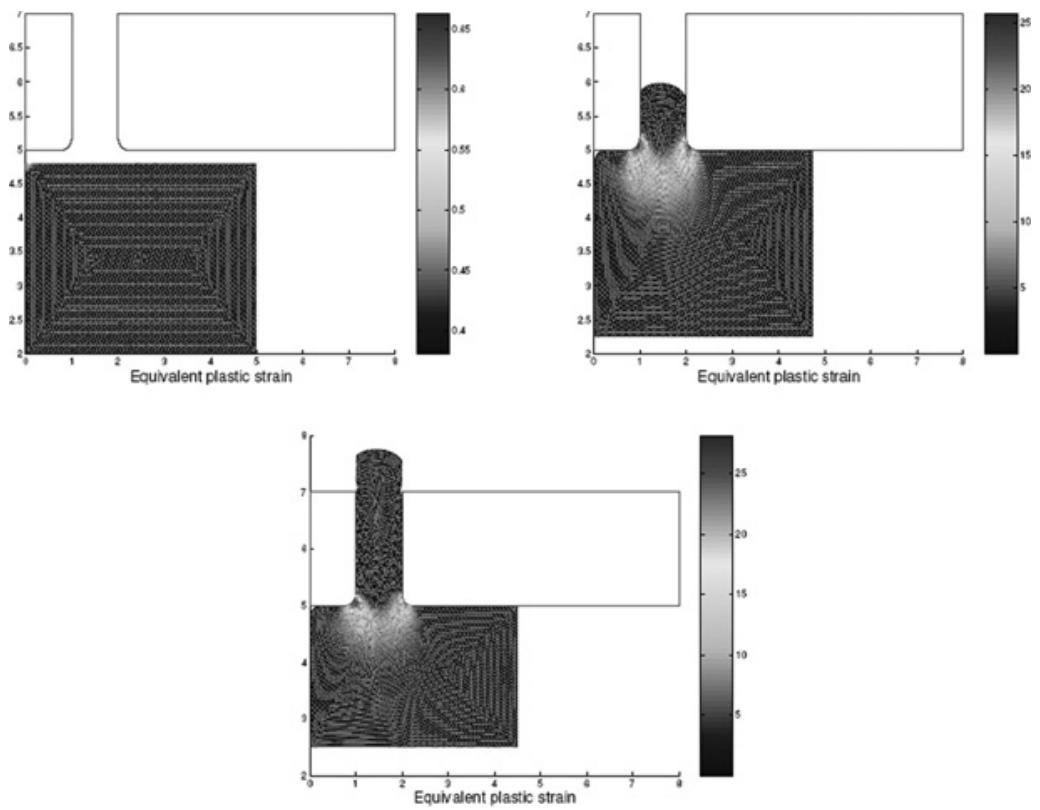

Figure 5.10. Equivalent plastic strain for time steps 1, 100 and 200. See Color Plate 2 on page 293.

In this example it can be seen how natural neighbour Galerkin methods constitute an appealing choice among numerical methods to simulate forming processes in general, and among meshless methods in particular. 


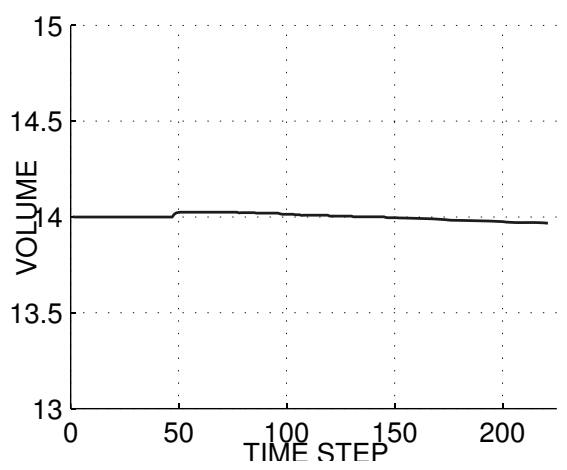

(a)

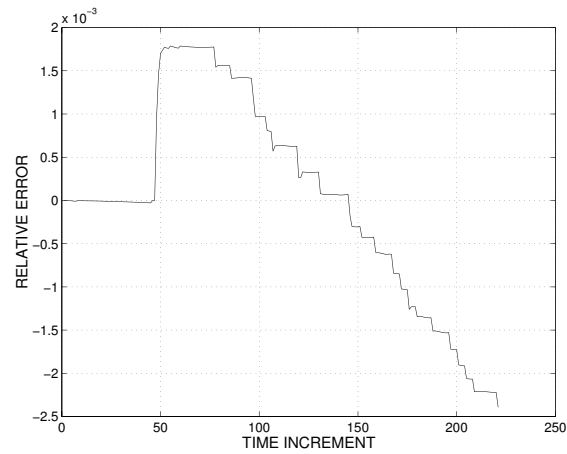

(b)

Figure 5.11. Volume of the computed $\alpha$-shape of the extruded metal (a) and associated relative error (b).

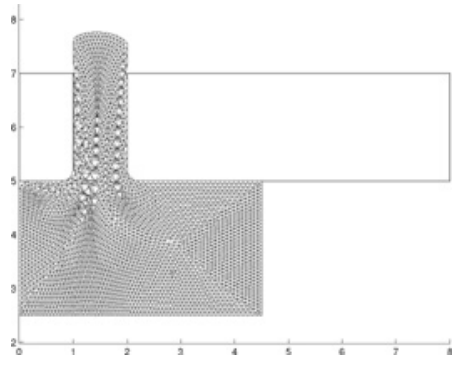

Figure 5.12. $\alpha$-shape of the cloud of points at the $200^{t h}$ time step.

\section{Closing Remarks}

In this paper we have briefly reviewed the characteristics of Natural Neighbour Galerkin methods. In particular, we have focused on the simulation of incompressible media and the development of stable mixed approximations through the use of Partition of Unity enrichment of Sibson interpolants.

Natural Neighbour Galerkin methods offer a sound geometrical basis for the treatment of free boundaries through the use of $\alpha$-shapes. It has been shown how the use of an appropriate $\alpha$-shape of the cloud of points allows us to accurately track the free surface of the domains through the simulated time interval. At the same time, the employ of $\alpha$-shapes ensures linear interpolation along the boundary of the domain. 
In summary, we believe that the $\alpha$-shape based Natural element Method $(\alpha-\mathrm{NEM})$ is an attractive choice when large distortion of the domain appears, both in Solid and Fluid Mechanics.

\section{References}

1. D. N. Arnold, F. Brezzi, and M. Fortin. A stable finite element for the Stokes equations. Calcolo, 21:337-344, 1984.

2. I. Babuška. The Finite Element Method with Lagrange multipliers. Numerische Mathematik, 20:179-192, 1973.

3. I. Babuška and J. M. Melenk. The partition of unity finite element method: Basic theory and applications. Comp. Meth. in Appl. Mech. and Eng., 4:289314, 1996.

4. K. J. Bathe. Finite Element procedures. Prentice-Hall, 1996.

5. J.-D. Boissonnat and F. Cazals. Natural neighbour coordinates of points on a surface. Computational Geometry: Theory and Applications, 19(2-3):155-173, 2001.

6. J. Bonet and S. Kulasegaram. Correction and stabilization of smooth particle hydrodynamics methods with applications in metal forming simulations. International Journal for Numerical Methods in Engineering, 47:1189-1214, 2000.

7. F. Brezzi. On the existence, uniqueness and approximation of saddle-point problems arising from lagrange multipliers. Revue Française d'Automatique Informatique Recherche Operationelle, Analyse Numérique, 8:129-151, 1974.

8. D. Chapelle and K. J. Bathe. The inf-sup test. Computers and Structures, 47(4-5):537-545, 1993.

9. E. Cueto, M. Doblaré, and L. Gracia. Imposing essential boundary conditions in the Natural Element Method by means of density-scaled $\alpha$-shapes. International Journal for Numerical Methods in Engineering, 49-4:519-546, 2000.

10. E. Cueto, N. Sukumar, B. Calvo, J. Cegoñino, and M. Doblaré. Overview and recent advances in Natural Neighbour Galerkin methods. Archives of Computational Methods in Engineering, 10(4):307-387, 2003.

11. B. Delaunay. Sur la Sphère Vide. A la memoire de Georges Voronoi. Izvestia Akademii Nauk SSSR, Otdelenie Matematicheskii i Estestvennyka Nauk, 7:793800, 1934.

12. H. Edelsbrunner, D. G. Kirkpatrick, and R. Seidel. On the shape of a set of points in the plane. IEEE Transactions on Information Theory, IT-29(4):551$559,1983$.

13. H. Edelsbrunner and E. Mücke. Three dimensional alpha shapes. ACM Transactions on Graphics, 13:43-72, 1994.

14. D. González, E. Cueto, and M. Doblaré. Volumetric locking in Natural Neighbour Galerkin methods. International Journal for Numerical Methods in Engineering, submitted for publication, 2003.

15. H Hiyoshi and K. Sugihara. Voronoi-based interpolation with higher continuity. In Proceedings of the 16th Annual ACM Symposium on Computational Geometry, pages 242-250, 2000.

16. R. W. Lewis, S. E. Navti, and C. Taylor. A mixed lagrangian-eulerian approach to modelling fluid flow during mould filling. International Journal for Numerical Methods in Engineering, 25:931-952, 1997. 
17. M. A. Martínez, E. Cueto, M. Doblaré, and F. Chinesta. Natural Element meshless simulation of injection processes involving short fiber suspensions. Journal of Non-Newtonian Fluid Mechanics, 115:51-78, 2003.

18. R. Sibson. A brief description of natural neighbour interpolation. In Interpreting Multivariate Data. V. Barnett (Editor), pages 21-36. John Wiley, 1981.

19. N. Sukumar, B. Moran, and T. Belytschko. The Natural Element Method in Solid Mechanics. International Journal for Numerical Methods in Engineering, 43(5):839-887, 1998.

20. M. Teichmann and M. Capps. Surface reconstruction with anisotropic densityscaled alpha shapes. In Proceedings of the 1998 IEEE Visualization Conference, 1998.

21. S. Timoshenko and J. N. Goodier. Teoría de la Elasticidad. Editorial Urmo, 1972.

22. G. M. Voronoi. Nouvelles Applications des Paramètres Continus à la Théorie des Formes Quadratiques. Deuxième Memoire: Recherches sur les parallélloèdres Primitifs. J. Reine Angew. Math., 134:198-287, 1908. 